\title{
Las agregaciones de desove de Tylosurus pacificus (Beloniformes: Belonidae) y su pesca en el Parque Nacional Natural Utría (Pacífico colombiano)
}

\author{
Tatiana Correa-Herrera ${ }^{1,2}$, Juan Diego Correa-Rendón ${ }^{1,2}$, Viviana Márquez-Velásquez ${ }^{1,3}$, \\ Luz Fernanda Jiménez-Segura $^{1}$ \& Juan David Carvajal-Quintero ${ }^{1,4}$ \\ 1. Universidad de Antioquia, Departamento de Biología Grupo de Ictiología (GIUA), Medellín, Colombia; \\ tatianacorreaherrera@gmail.com,jdiego.correa@udea.edu.co,luz.jimenez@udea.edu.co \\ 2. Universidad de Antioquia, Corporación Académica Ambiental, Grupo de Estudios Oceánicos (GEOc). \\ 3. Centro de ciências exatas e da natureza, Universidade Federal da Paraíba, João Pessoa- PB, Brasil; \\ viviomar@gmail.com \\ 4. Red de Ecoetología, Instituto de Ecología, Veracruz, México; juanchocarvajal@gmail.com
}

Recibido 09-XII-2015. Corregido 26-IX-2016. Aceptado 25-X-2016.

\begin{abstract}
Spawning aggregations of Tylosurus pacificus (Beloniformes: Belonidae) and their fishery in Utría Park, Colombian Pacific. Some species of marine fish have complex relationships with coastal ecosystems during their reproductive periods, as the needle fish Tylosurus pacificus that forms spawning aggregations in gravel beaches in Utría Park. We described fishery and some aspects of the reproductive biology of T. pacificus and proposed some strategies to mitigate the impact of this event and associated ecological processes. Fisheries monitoring were conducted between April 2008 and February 2009 in the Utría National Park, Colombian Pacific. Catch volumes, length structure, sex ratio and fecundity $(n=84)$ were analyzed. The total length ranged among 60.5 and $104 \mathrm{~cm}$ with a mean and standard deviation of $85.35 \pm 9.09 \mathrm{~cm}$. The average fertility of oocytes per gonad was 189 685.56, with a mode of $8 \mathrm{~mm}$ diameter and an asynchronous gonadal development. This species spawns collectively on the beach when the tide reaches the highest level during full and new moon, usually in the evening. Fishermen of a near town take advantage of the spawning aggregation of this species. The reproduction study of T. pacificus and ecological processes associated with their spawning, should be expanded to give adequate use, and ensure the sustainability of their fishery over the long term. Rev. Biol. Trop. 65 (1): 77-87. Epub 2017 March 01.
\end{abstract}

Key words: needlefish, spawning, fecundity, reproduction, Utría Park, Colombian Pacific.

Los miembros de la familia Belonidae se conocen como peces aguja, sus especies se distribuyen en zonas marinas templadas y tropicales, y algunos géneros estan restringidos a ecosistemas dulceacuícolas (Nelson, 2006). Los Belónidos marinos presentan hábitos epipelágicos oceánicos y costeros, y pueden alcanzar hasta $2 \mathrm{~m}$ de longitud total (Collette, 2003). Las especies costeras son ovíparas y depositan un gran número de huevos grandes con filamentos coriónicos típicamente largos que les permite adherirse al sustrato. Estos huevos son generalmente más grandes que en otros Beloniformes (2.3 a $4.3 \mathrm{~mm}$ de diámetro). La gónada izquierda tiende a estar reducida en longitud o incluso perderse en algunas especies, particularmente en las hembras (Collette, McGowen, Parin \& Mito, 1984).

En general, la información biológica de los Belónidos ha sido documentada, aunque algunas especies de esta familia han sido poco estudiadas. Este es el caso de la aguja ensenadeña Tylosurus pacificus (Steindachner, 1876). Las investigaciones realizadas sobre esta especie se concentran en aspectos taxonómicos y de su distribución geográfica (Collette, 1995; Lovejoy, 2000; Collette \& Banford, 2001; Williams \& Bunkley-Williams, 2005; Rojas \& Zapata, 
2006; Tobón, Rubio, \& Giraldo, 2008; BernalCastro, 2008; Castellanos-Galindo, Krumme, \& Willis, 2010; Collette, Acero, \& Rojas, 2010; Palacios-Salgado, Moreno-Sánchez, AbitiaCárdenas, \& Gutiérrez-Sánchez, 2012). Estos trabajos afirman que T. pacificus es una especie epipelágica que puede encontrarse también en áreas costeras (Collette, 1995), y que esta ampliamente distribuida en el Pacífico Oriental, desde México hasta Perú, incluyendo el sur de Baja California, el Golfo de California central y la Isla del Coco y Galápagos (Collette, Acero, \& Rojas, 2010). Adicionalmente, se ha reportado que $T$. pacificus es de interés pesquero en algunas zonas del Pacífico Oriental (Collette, 1995).

En el Parque Nacional Natural Utría (PNNU) en el Pacífico Colombiano, esta especie es explotada con fines de consumo local. Las autoridades del Parque permiten la pesca artesanal con el uso de artes de pesca consideradas selectivas como la línea de mano, esto con la intención de minimizar el impacto social y cultural sobre las comunidades que ancestralmente han venido realizando estas prácticas extractivas en el área (Unidad Administrativa Especial del Sistema de Parques Nacionales Naturales, 1998). Los registros obtenidos en 2008 resaltan la importancia de la actividad pesquera en el PNNU, ya que se registraron 11.8 toneladas en ese año (Correa-Rendón, Palacio, \& Jiménez-Segura, 2012). Sin embargo, en los últimos años T. pacificus o la aguja ensenadeña como se le conoce localmente, ha sido objeto de una fuerte presión de pesca ilegal por parte de algunos grupos de pescadores que habitan las zonas aledañas al PNNU. Estas comunidades aprovechan para realizar las capturas en el momento en el que los individuos de esta especie se acercan a desovar en las playas del área protegida. Esta situación ha llevado a que las autoridades ambientales de Colombia la cataloguen como una especie en categoría de riesgo en la región del Pacífico Colombiano (UAESPNN, 2007). A pesar de la prioridad de conservación que presenta esta especie, el desconocimiento de su biología no ha permitido desarrollar estrategias específicas para su preservación. Este trabajo tiene como propósito proveer información sobre algunos aspectos pesqueros y reproductivos de $T$. pacificus, siendo la primera caracterización de la pesca, desove y fecundidad que se realiza para esta especie.

\section{MATERIALES Y MÉTODOS}

Área de estudio: El PNNU se encuentra en el Pacífico oriental tropical (Fig. 1), una de las zonas más inexploradas y aisladas en el mundo (Robertson, Grove, \& McCosker, 2004). Está ubicado al norte del Pacífico Colombiano (5'52'6.9” N - 7708'77.24” W) $\mathrm{y}$ tiene una extensión total (marina y terrestre) de 54300 ha (UAESPNN-Dirección Territorial Noroccidente, 2006). Esta zona es parte de la región denominada Provincia Biogeográfica del Chocó, considerada como de alta prioridad de conservación a nivel mundial debido a su diversidad biológica y nivel de endemismos (Myers, Mittermeier, \& Mittermeier, 2000). Dentro del área marina del PNNU se encuentra la Ensenada de Utría, cuerpo de agua con dirección Norte-Sur. Tiene una longitud de $6 \mathrm{~km}$, un ancho medio de $800 \mathrm{~m}$ y se encuentra rodeada por montañas de poca elevación (UAESPNN, 1998). Estas elevaciones montañosas están cubiertas por selva húmeda tropical y en el borde marino de la zona nororiental de la ensenada, por franjas angostas de manglar (Von Prahl, Cantera, \& Contreras, 1990). El área protegida posee un clima cálido superhúmedo, con una temperatura entre 27 y $30{ }^{\circ} \mathrm{C}$, una humedad relativa de $90 \%$ (Eslava, 1995) y una precipitación que supera $6100 \mathrm{~mm}$ de lluvia en promedio multianual (UAESPNN, 2007). La marea es de tipo semidiurno, el cambio en la altura entre la bajamar y la pleamar puede sobrepasar los $4 \mathrm{~m}$. El agua de la Ensenada es cálida y allí se presenta gran diversidad de ecosistemas marinos (ie. manglares, corales, litorales rocosos y playas de arena y grava) y esta condición la convierte en un lugar propicio para la llegada de especies migratorias y el desove de peces como T. pacificus. 


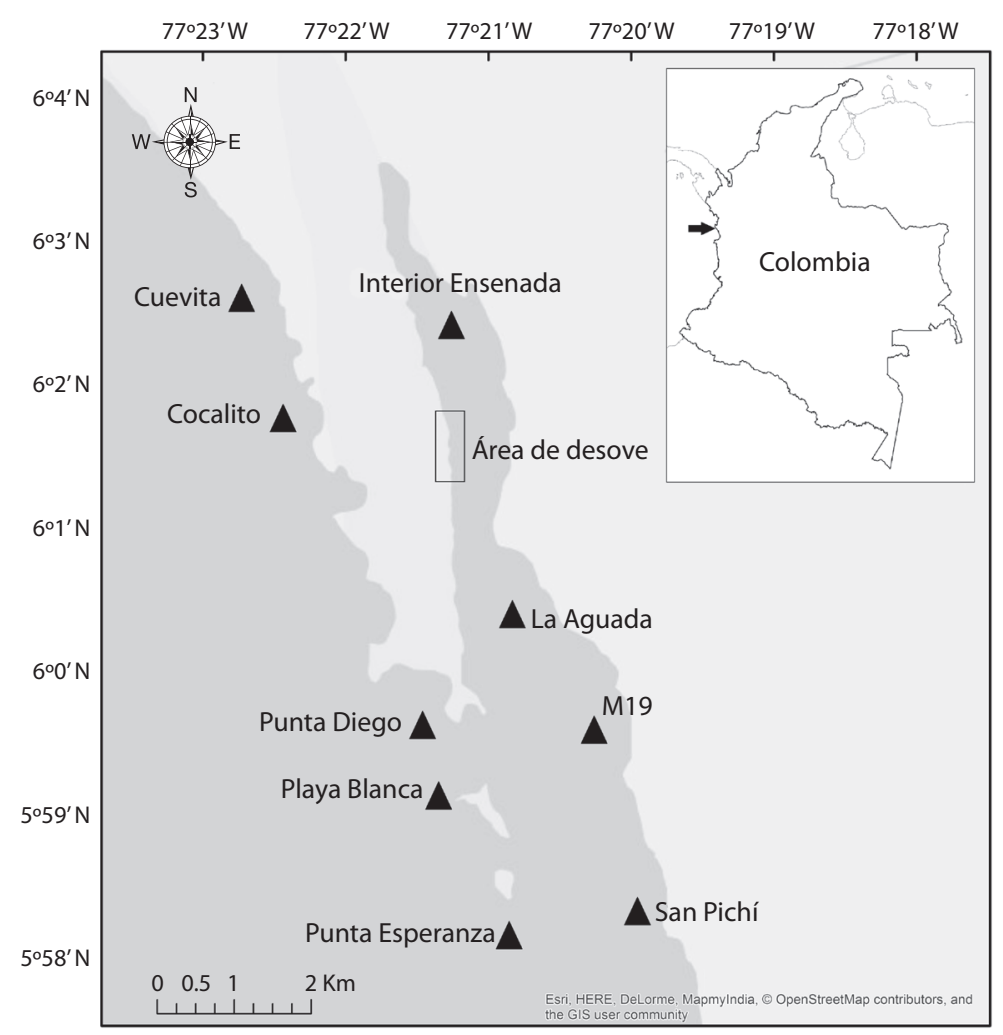

Fig. 1. Área de estudio. Caladeros de pesca en el Parque Nacional Natural Utría.

Fig. 1. Study area. Fishing grounds within the marine area of Utría Park.

\section{Captura de $T$. pacificus en la pesca arte-} sanal: Entre abril 2008 y febrero 2009 se registraron diariamente los desembarcos de la pesca artesanal de 21 unidades de pesca, cuya actividad se concentró en diez caladeros ubicados al interior del PNNU (Fig. 1). Todas las capturas registradas fueron realizadas por medio de arrastres superficiales de línea de mano usando como carnada viva a Selar crumenophthalmus (Bloch, 1793) y Eucinostomus currani (Zahuranec, 1980). El trabajo se realizó con la participación concertada de pescadores de la zona. La captura en número de individuos se contabilizó para cada mes y caladero. Se midió la longitud total $( \pm 0.1 \mathrm{~cm})$ de cada ejemplar de T. pacificus con un ictiómetro de metal de 150 cm y se pesó ( $\pm 0.1 \mathrm{~g})$ con una báscula de reloj. Además, con base en la información obtenida durante el ciclo anual, se estimó la abundancia relativa mensual de la especie. Para esta caracterización pesquera, no se tuvo en cuenta la pesca realizada por parte de las comunidades locales durante los eventos reproductivos de $T$. pacificus, debido a que no fue posible concertar con los pescadores el monitoreo de sus capturas, posiblemente porque este tipo de pesca es considerada ilegal por las autoridades ambientales regionales.

Aspectos reproductivos de $T$. pacificus: Durante el monitoreo pesquero, el sexo de cada ejemplar fue reconocido según la forma de las gónadas y los productos sexuales. Para verificar si la proporción de sexos era diferente de la esperada (M:H: 1:1), se utilizó la prueba Chi-cuadrado. Las gónadas fueron analizadas macroscópicamente siguiendo a Vazzoler (1996) quien propone cinco categorías: A (inmaduro), B (adulto madurando), C (adulto maduro), D (adulto desovado) 
y E (adulto en reposo). La fecundidad fue estimada a partir del conteo de ovocitos de siete gónadas de hembras maduras. El número de ovocitos fue estimado empleando el método gravimétrico (Vazzoler, 1996). Cada gónada fue pesada en una balanza con una precisión de $0.01 \mathrm{~g}$, del total de la gónada se extrajo una submuestra de $1 \mathrm{~g}$ de la región anterior, media y posterior; en cada sub-muestra fue contado el número de ovocitos con la ayuda de un estereoscopio. Para definir el tipo de desove, se tomaron aleatoriamente 100 ovocitos de cada submuestra y se midió su diámetro con la ayuda de una reglilla ocular de un estereoscopio Olympus SZ-ST.

El desove de $T$. pacificus y su pesca durante este evento: Se acompañó la actividad pesquera de la aguja ensenadeña durante su desove en las playas reconocidas por los pescadores para este fin. Se realizaron entrevistas no estructuradas a los pescadores para describir el desove de la especie y las artes de pesca usadas para capturarla durante este evento. Después del desove de la aguja ensenadeña ocurrido el 4 de noviembre de 2008, se realizó dos veces por semana el seguimiento visual de los huevos depositados en las playas, hasta que fueron retirados 15 días después por la pleamar durante la marea viva o de sicigia.

\section{RESULTADOS}

Captura de T. pacificus en la pesca artesanal: Durante los 11 meses de monitoreo de la pesca artesanal se registraron 84 individuos y una biomasa total de 81910 g. Entre las capturas se registraron individuos con pesos entre 300 y 2300 g (media $\pm \mathrm{SD}=970 \pm 380 \mathrm{~g}$ ) y longitudes totales entre 60.5 y $104 \mathrm{~cm}$ (media $\pm \mathrm{SD}=85.3 \pm 9.1 \mathrm{~cm}$ ). Los meses que presentaron las mayores capturas en número fueron julio $(53.57 \%)$ y septiembre $(26.79 \%)$ así como de biomasa (37.17\% y $20.72 \%$ respectivamente) (Fig. 2). La mayoría de las capturas en número de individuos y biomasa se registraron en los caladeros Punta Esperanza (42.86 \% y $35.12 \%)$ y en La Aguada (20.24 \% y 17.83 $\%$ ), que corresponden a arrecife rocoso y zona coralina, respectivamente.

Aspectos reproductivos de $T$. pacificus: Se capturaron ejemplares maduros (estadio C) durante la mayor parte del periodo de estudio, excepto en abril y junio. En julio y noviembre se presentó el mayor porcentaje de individuos maduros (30.36 y $16.07 \%$ respectivamente).

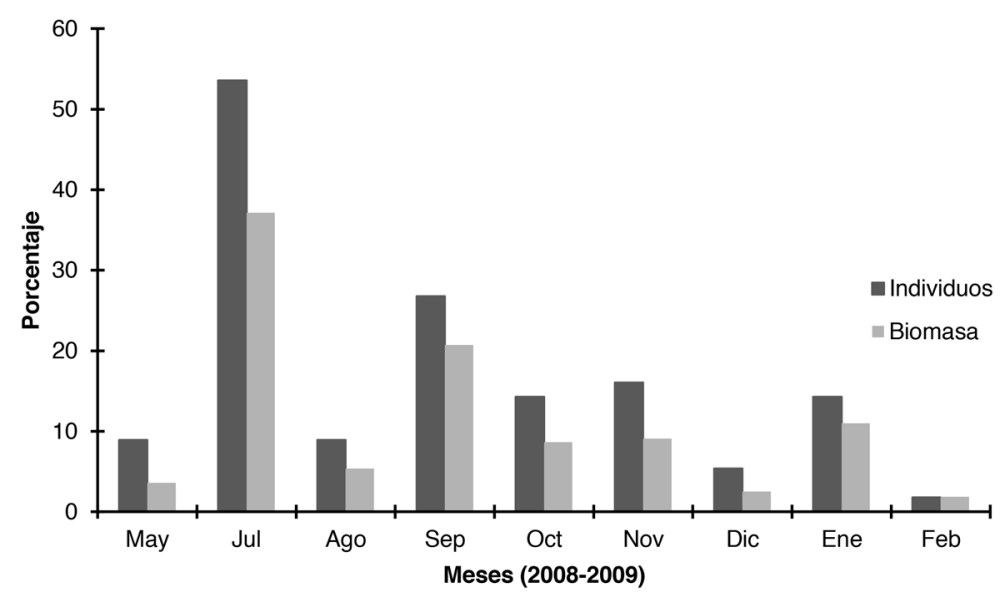

Fig. 2. Distribución mensual del número de individuos y biomasa de Tylosurus pacificus capturada por los pescadores en el PNN Utría entre abril 2008 y febrero 2009 ( $n=84)$.

Fig. 2. Monthly distribution of the number of individuals and biomass of Tylosurus pacificus captured by fishermen in Utría Park between April 2008 and February 2009 (n=84). 
Los individuos de menor talla capturados $(60.5$ $\mathrm{cm})$ también se encontraron maduros. La aguja ensenadeña presentó una proporción de sexos ajustada a la esperada (H:M; $\mathrm{n}_{\text {machos }}=40 ; \mathrm{n}_{\text {hem }}$ bras $\left.=33 ; X^{2}=10.797, \mathrm{p}>0.05\right)$. Los machos se capturaron principalmente entre los 70 y $90 \mathrm{~cm}$ longitud total (Lt) y las hembras entre los 80 y $100 \mathrm{~cm} \mathrm{Lt} \mathrm{(Fig.} \mathrm{3).} \mathrm{Se} \mathrm{estimó} \mathrm{una}$ fecundidad promedio de 189685.56 ovocitos por gónada, con un diámetro promedio de 8.6 mm para ejemplares entre 86 y $104 \mathrm{~cm}$ Lt.
Se identificaron dos grupos en la distribución de frecuencia del diámetro de los ovocitos, el primer grupo con los mayores diámetros pero con las menores frecuencias, y el segundo grupo con los menores diámetros y mayores frecuencias (Fig. 4).

El desove de $T$. pacificus y su pesca durante este evento: La aguja ensenadeña desova en el PNNU durante las mareas vivas que suceden cada 15 días (conocidas localmente

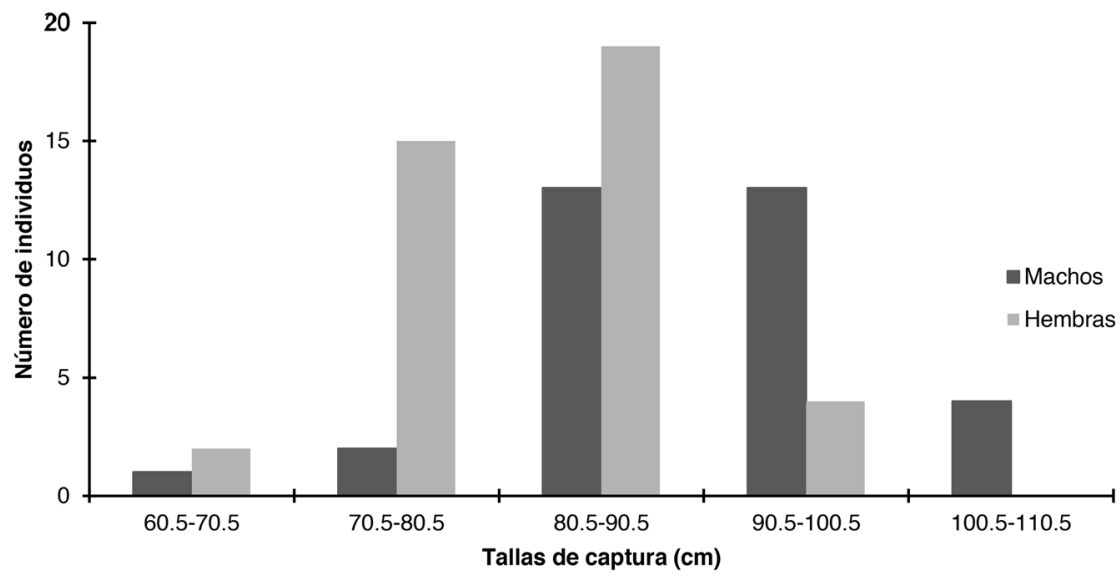

Fig. 3. Proporción de sexos según la talla de Tylosurus pacificus en el PNN Utría entre abril 2008 y febrero 2009. Hembras en gris y machos en negro $(n=73)$.

Fig. 3. Sexual proportion by size of Tylosurus pacificus in Utría Park between April 2008 and February 2009. Females in gray and Males in black $(\mathrm{n}=73)$.

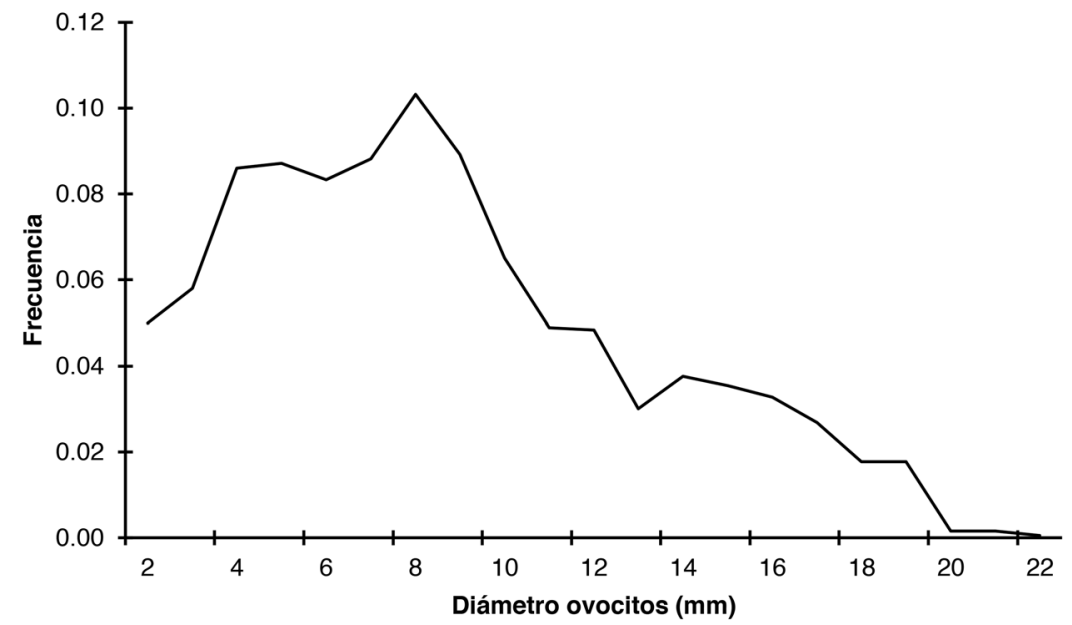

Fig. 4. Frecuencia del diámetro de los ovocitos de Tylosurus pacificus en el PNN Utría ( $\mathrm{n}=700)$.

Fig. 4. Oocyte diameter frequency of Tylosurus pacificus in Utría Park $(\mathrm{n}=700)$. 
como pujas), cuando la pleamar alcanza su máxima altura entre 3.5 y $4 \mathrm{~m}$. El desove ocurre generalmente al anochecer, en ese momento, los peces se acercan en forma colectiva a la playa para liberar los productos sexuales y este comportamiento hace que sean de fácil captura. Las playas donde se dan estos desoves se caracterizan por estar compuestas por grava y tienen gran estabilidad, ya que es escasa su renovación natural. Los pescadores que habitan en El Valle (corregimiento del municipio Bahía Solano, Chocó) conocen las agregaciones de desove realizadas por la aguja ensenadeña y se desplazan al PNNU para pescarla. La estrategia de pesca consiste en concentrarse en las playas reconocidas tradicionalmente como áreas de desove en tramos de $20 \mathrm{~m}$ de longitud aproximadamente (Fig. 1). Para capturar la aguja emplean ganchos y anzuelos (Fig. 5) que lanzan al agua y recobran rápidamente. Los peces atrapados son acumulados en las embarcaciones que permanecen en tierra firme durante la faena de pesca. Muchos de esos individuos no logran desovar antes de la captura y lo hacen en las embarcaciones (Fig. 5). Dado que la pesca de esta especie transcurre en las horas de la noche, los funcionarios que administran el área protegida no la detectan. Adicional a estas jornadas de captura con anzuelos, los pescadores usan trasmallo para capturar
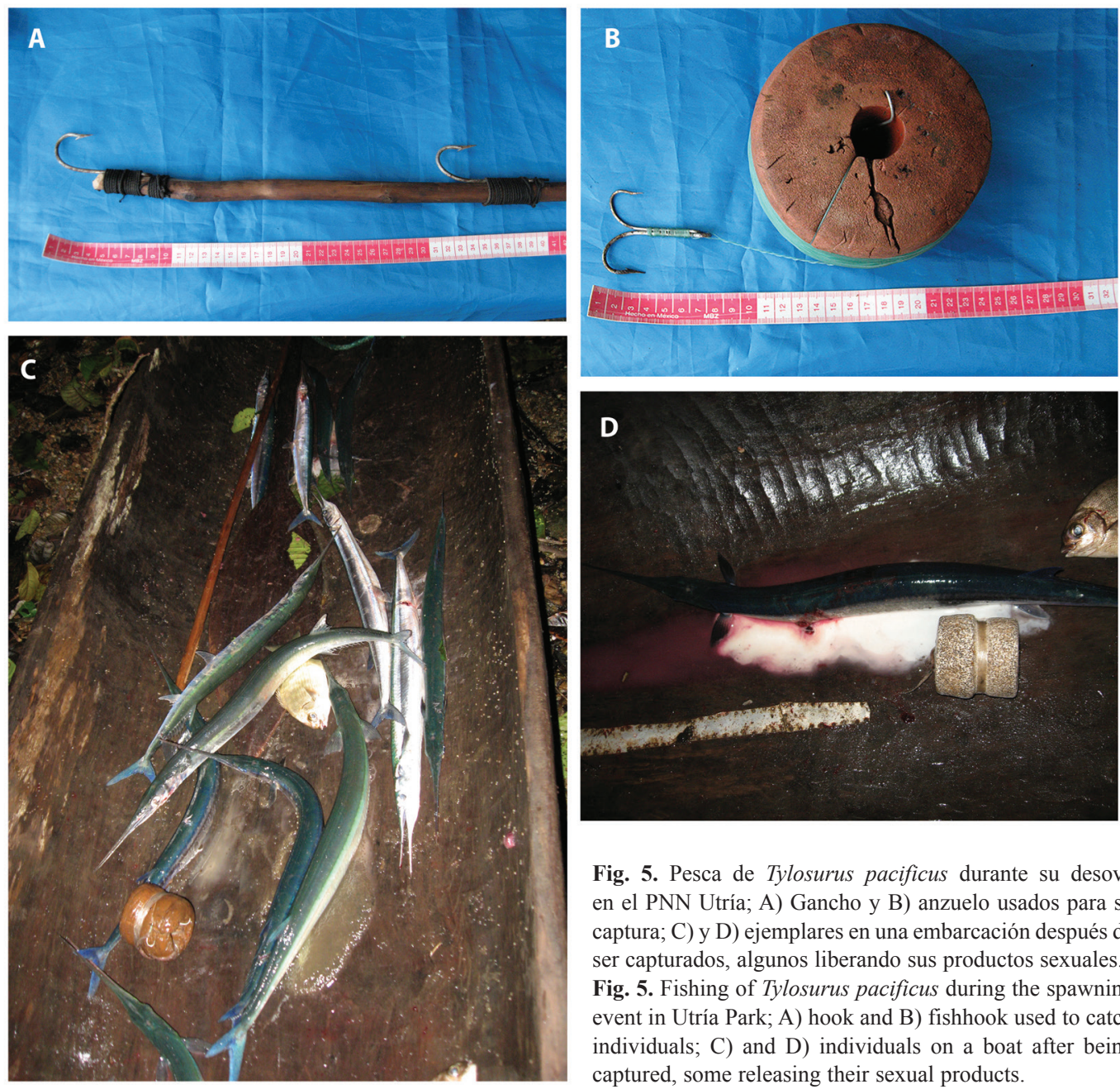

Fig. 5. Pesca de Tylosurus pacificus durante su desove en el PNN Utría; A) Gancho y B) anzuelo usados para su captura; C) y D) ejemplares en una embarcación después de ser capturados, algunos liberando sus productos sexuales.

Fig. 5. Fishing of Tylosurus pacificus during the spawning event in Utría Park; A) hook and B) fishhook used to catch individuals; C) and D) individuals on a boat after being captured, some releasing their sexual products. 
aquellas agujas que no se acercan a la orilla, así como otras especies de peces que se hallan asociadas al evento reproductivo; tal vez en busca de alimento. Los huevos de T. pacificus depositados en las playas del PNNU forman tapetes que permanecen durante 15 días fuera del agua hasta la siguiente marea viva. Durante el tiempo que los huevos permanecieron en la playa, se apreciaron cambios en su coloración a medida que el embrión se desarrollaba, tomando un color naranja oscuro en su fase más avanzada, en la que se observó el movimiento del embrión al interior del huevo (Fig. 6).

\section{DISCUSIÓN}

Las capturas de $T$. pacificus en el PNNU no fueron representativas en los desembarcos de la pesca artesanal en términos de biomasa y número de individuos, sin embargo, no se conoce con certeza la magnitud de la pesca de la especie durante los eventos de desove. Esta pesca es considerada ilegal y esta prohibida en el PNNU, por lo cual no fue posible cuantificar estas capturas. Tobón et al. (2008) registraron en el Golfo de Tribugá a T. pacificus como una especie capturada ocasionalmente, incluso a nivel de familia se reportó un bajo aporte numérico y en biomasa. Según Escobar (2010) esta especie es considerada a nivel local como una de las más apetecidas por el sabor de su carne.

La variación en la captura de la especie puede originarse por múltiples factores que van desde oscilaciones en su reclutamiento natural, movimientos a pequeña, mediana y gran escala (p.e. movimientos de forrajeo o territoriales y migraciones) o bien a la variabilidad en el esfuerzo pesquero (Dingle, 1996). Estas son hipótesis que podrían ser verificadas en estudios futuros. Hábitats como arrecifes rocosos y zonas coralinas son sitios de preferencia de $T$. pacificus. Estos hábitats son estructuras complejas (Little \& Kitching, 1996) que albergan una alta diversidad de peces (Gómez \& Vieira, 1996). Dicha condición representa una alta oferta de presas y de refugio para muchas especies de peces, incluso durante la temporada de
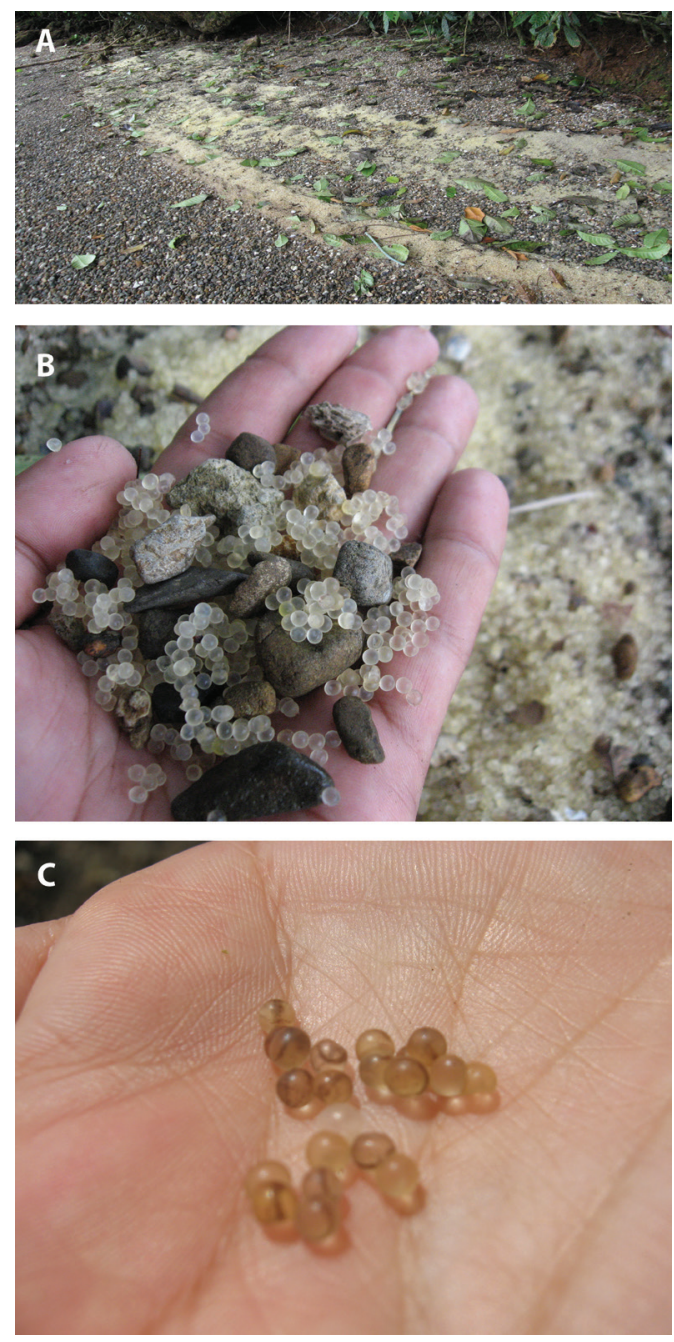

Fig. 6. Huevos de Tylosurus pacificus en la playa de desove en el PNN Utría, A) huevos ovopositados en una playa de grava en la ensenada de Utría; B) huevos en una fase temprana de desarrollo; C) huevos en una fase avanzada de desarrollo en la que puede apreciarse el embrión.

Fig. 6. Eggs of Tylosurus pacificus on the spawning beach in Utría Park, A) eggs laid on a gravel beach in Utría inlet; B) eggs in an early stage of development; C) eggs in an advanced stage of development in which the embryo can be seen.

reproducción (Estupiñán, Von Prahl, \& Rubio, 1990; Bellwood \& Wainwright, 2002).

La proporción de sexos determinada para T. pacificus en el PNNU (1:1) sugiere que no hay una segregación sexual de la población. Asimismo, se ha demostrado que la proporción 
de sexos de cada especie, presenta caracteres propios, esta sujeta a gran variabilidad y que evidentemente este factor tiene un carácter adaptativo específico en relación con la reproducción, pero no siempre se obtiene una representación real de esta relación debido a que la proporción sexual esta sujeta con frecuencia, a diferencias en el hábitat de cada sexo, lo cual puede variar durante el ciclo anual y durante la ontogenia (García, Claro, \& Koshelev, 1994; Longart, Acosta, Parra, \& Lista, 2012; CorreaHerrera \& Jiménez-Segura, 2013).

La fecundidad de una especie es el resultado de un proceso evolutivo en el que se optimiza el reparto de energía obtenida por la alimentación entre el consumo metabólico, el crecimiento y la reproducción y tiene un carácter adaptativo (Saborido-Rey, 2004). La fecundidad de T. pacificus puede considerarse alta si se le compara con lo reportado para otras especies del género. Tylosurus acus (promedio de 25629 ovocitos, Liao \& Chang, 2011) y T. fodiator (promedio de 53605 ovocitos, Escobar, 2010). Debido a su alta fecundidad y frecuentes desoves, se presume que T. pacificus sigue una estrategia tipo $r$, la cual le permitiría sobrevivir en cuanto se dan condiciones ambientales favorables (Saborido-Rey, 2004).

La presencia de dos grupos en la distribución de frecuencia del diámetro de los ovocitos de T. pacificus indica el desarrollo asincrónico de los mismos y sugiere que desova grupos de ovocitos en diferentes periodos del año. De acuerdo a Wallace y Selman (1981) este tipo de distribución de los ovocitos es característico dentro de las estrategias de múltiples desoves anuales, con desarrollo de los ovarios sincrónico por grupos. Resultados similares han sido reportados para T. fodiator por Escobar (2010) también en el PNNU y para T. acus por Liao y Chang (2011). Estos desoves fraccionados y períodos prolongados de desove son característicos de especies de peces tropicales y subtropicales, no sólo como una adaptación a las fluctuaciones en la disponibilidad de alimento, sino para la preservación de las especies bajo condiciones desfavorables al desove (Nikolsky, 1963).
Las artes de pesca usadas en el PNNU capturan los ejemplares de T. pacificus por encima de su talla media de madurez sexual, ya que los individuos más pequeños capturados $(60.5 \mathrm{~cm})$ se encontraron maduros. La mayoría de las capturas se presentaron en julio, cuando la mayoría de los individuos estaban maduros. Este hecho podría estar ejerciendo presión sobre esta población, por lo que son necesarios estudios complementarios sobre otros aspectos biológicos como edad y crecimiento, ciclos reproductivos, determinación de tallas de madurez sexual, uso del hábitat, entre otros, para evaluar los posibles efectos sobre la población. La investigación pesquera junto con información sobre la biología de especies sujetas a explotación, es relevante para regular la actividad durante las temporadas de reproducción y así mantener la capacidad de una especie de reclutar individuos jóvenes al ambiente, asegurando de este modo la supervivencia de la especie y su pesquería en una zona (Castello, Sunye, Haimovici, \& Hellebrandt, 2009).

Se ha encontrado que la dinámica reproductiva de $T$. pacificus esta asociada con el periodo lunar y máximo nivel de la pleamar, lo que permite que los peces puedan acceder a la zona más alta de la playa de grava para depositar los huevos. Estos huevos presentan filamentos cortos que ayudan a mejorar su adherencia al sustrato (Bernal-Castro, 2008). La alta pendiente de las playas también facilita el acceso de la especie a la zona superior en pleamar. Yáñez-Arancibia (1986) plantea que las playas de grava en el Pacífico tropical se caracterizan por presentar sedimentos no consolidados, con una inclinación pronunciada entre 10 y 20 grados, con numerosas bermas creadas por las olas a lo largo de la parte superior de la playa, donde el suministro natural de los sedimentos es el más lento de todos los tipos de playas. Esta lenta renovación de los sedimentos le permite a los huevos permanecer en el mismo lugar durante el desarrollo del embrión y no quedar a la deriva en aguas someras donde fácilmente serían depredados (Bernal-Castro, 2008).

En Micronesia se han reportado agregaciones reproductivas de peces aguja asociados 
con eventos lunares y de mareas, tales como máximas mareas (Rhodes, 2003). Así mismo, Bernal-Castro (2008) menciona que la dinámica reproductiva del $T$. pacificus en Costa Rica obedece a un patrón de agregaciones residente, que ocurre cada mes. En el PNNU, los pescadores indican que estos eventos de desove suceden quincenalmente, asociados a las mareas vivas.

La actividad pesquera asociada con estos eventos reproductivos no ha sido estudiada en el PNNU y no hay acciones de control al respecto a pesar de considerarse una pesca ilegal. Este estudio espera tener un impacto positivo en la protección de la aguja ensenadeña y estimular el desarrollo de investigaciones adicionales sobre la mortalidad de los embriones debido a la depredación y a la destrucción mecánica de los huevos por el pisoteo generado por los pescadores. Otro aspecto de atención es el desconocimiento de los procesos ecológicos asociados a los eventos reproductivos de la aguja ensenadeña. Es de suponer que éstos juegan un papel importante en el flujo de energía en las redes tróficas; no solo en el área marina sino también en la franja costera. Otras especies de peces depredadores aprovechan las agregaciones reproductivas de T. pacificus para alimentarse, no solo de los huevos sino también de los individuos desovantes. La drástica reducción o extirpación de agregaciones de desove ciertamente afectará el desarrollo y la salud de las comunidades biológicas asociadas, pero pueden esperarse impactos ecológicos más amplios.

De acuerdo con estos resultados, es indispensable dar inicio a estudios reproductivos detallados, para poder dar un manejo adecuado a la pesca de la especie y garantizar la sostenibilidad de su pesquería en el largo plazo. Hacer un seguimiento de la pesca durante los desoves para conocer la talla media de madurez, corroborar los datos de la fecundidad y estimar la cantidad de huevos que se estan dejando de ovopositar, además de estudiar la sobrevivencia de los huevos mientras permanecen en la playa y conocer sus depredadores tanto en tierra como en el agua. Es de interés para la protección de la población de Tylosurus pacificus que el PNNU restrinja la pesca en las áreas coralinas y desestimule la pesca de la aguja ensenadeña durante sus eventos de desove. La inclusión de los pescadores dentro de actividades de conservación y en el empoderamiento de sus recursos puede constituir una estrategia de conservación válida y efectiva en el mediano y largo plazo. Después de conocer con más detalle la dinámica de los eventos de desove podría establecerse al menos un periodo de veda en el cual se protejan algunos desoves en el año, (potencialmente en julio y noviembre, cuando se encontraron la mayor cantidad de ejemplares maduros) y definir una cuota pesquera de manera que los pescadores no pierdan su tradición cultural y puedan seguir accediendo al recurso.

\section{AGRACECIMIENTOS}

Agradecemos a la Unidad de Parques Nacionales Naturales de Colombia-Región Occidente, en especial a las directivas del Parque Nacional Natural Utría y a sus funcionarios en campo, a Patrimonio Natural por el apoyo financiero, a la Universidad de Antioquia (en especial a los grupos de investigación GIUA y GAIA) por su apoyo financiero, logístico $\mathrm{y}$ académico $\mathrm{y}$, muy especialmente a los pescadores y la comunidad del Valle, Jurubidá y Bahía Solano, sin cuya colaboración no hubiese sido posible obtener la información utilizada en esta investigación.

\section{RESUMEN}

Las agregaciones de desove de Tylosurus pacificus (Beloniformes: Belonidae) y su pesca en el Parque Nacional Natural Utría (Pacífico colombiano). Algunas especies de peces marinos tienen complejas relaciones con los ecosistemas costeros durante sus periodos reproductivos, tal es el caso del pez aguja Tylosurus pacificus, que conforma agregaciones de desove en playas de grava en el Parque Nacional Natural Utría. Se describe la actividad pesquera y algunos aspectos de la biología reproductiva de T. pacificus y se proponen algunas medidas para mitigar el impacto sobre este evento y los procesos ecológicos asociados. Se realizó un monitoreo pesquero entre abril 2008 y febrero 2009 en el Parque Nacional Natural Utría (PNNU), 
Pacífico colombiano. Se analizaron los volúmenes de captura, estructura por talla, proporción de sexos y fecundidad $(\mathrm{n}=84)$. La longitud total osciló entre $60.5 \mathrm{~cm}$ y $104 \mathrm{~cm}$ con una media y desviación estándar de $85.35 \pm 9.09 \mathrm{~cm}$. La fecundidad promedio fue 189685.56 ovocitos por gónada, con una moda de $8 \mathrm{~mm}$ de diámetro y un desarrollo gonadal asincrónico. La especie desova en forma colectiva en la playa cuando la marea alcanza el nivel más alto durante la luna llena y nueva, generalmente al anochecer. Pescadores que habitan los pueblos cercanos aprovechan este recurso, durante la agregación reproductiva de la especie. Es importante ampliar el estudio de la reproducción de T. pacificus y los procesos ecológicos asociados a su desove para poder dar un aprovechamiento adecuado y garantizar la sostenibilidad de su pesquería a largo plazo.

Palabras clave: pez aguja, desove, fecundidad, reproducción, PNN Utría, Pacífico colombiano.

\section{REFERENCIAS}

Bellwood, D. R., \& Wainwright, P. C. (2002). The history and biogeography of fishes on coral reefs. In P. F. Sale (Ed.), Coral Reef Fishes. Dynamics and diversity in a complex ecosystem (pp. 5-32). San Diego: Academic Press.

Bernal-Castro, M. P. (2008). Caracterización del desove del pez aguja. Tylosurus sp. (Orden: Beloniformes, Familia: Belonidae) en el golfo Dulce, península de Osa (Trabajo de Grado). Universidad Nacional de Costa Rica, Costa Rica.

Castellanos-Galindo, G. A., Krumme, U., \& Willis, T. J. (2010). Tidal influences on fish distributions on tropical eastern Pacific rocky shores Colombia. Marine Ecology Progress Series, 416, 241-254.

Castello, J. P., Sunye, P. S., Haimovici, M., \& Hellebrandt, D. (2009). Fisheries in southern Brazil: A comparison of their management and sustainability. Journal of Applied Ichthyology, 25, 287-293.

Collette, B. B. (2003). Family Belonidae Bonaparte 1832: Needle- fishes. Proceedings of the California Academy of Sciences, Annotated Checklists of Fishes, $16,1-22$.

Collette, B. B. (1995). Belonidae. Agujones, maraos. In W. Fischer, F. Krupp, W. Schneider, C. Sommer, K. E. Niem, \& V. H. Carpente (Eds.), Guía FAO para la Identificación de especies para los fines de la pesca. Pacífico Centro-Oriental (pp. 919-926). Roma: FAO.

Collette, B., Acero, A., \& Rojas, P. (2010). Tylosurus pacificus. En The IUCN Red List of Threatened Species 2010. Recuperado de http://www.iucnredlist.org/

Collette, B. B., Acero, A., \& Rojas, P. (2010). Tylosurus pacificus. En IUCN 2013. IUCN Red List of Threatened Species. Version 2015- 4. Reviewed from http://: www.iucnredlist.org
Collettte, B. B., \& Banford, H. M. (2001). Status of the eastern Pacific agujon needlefish Tylosurus pacificus (Steindachner, 1876) (Beloniformes: Belonidae). Revista de Biología Tropical, 49(1), 51-57.

Collette, B. B., McGowen, G. E., Parin, N. V., \& Mito, S. (1984). Beloniformes: development and relationships. In H. G. Moser, W. J. Richards, D. M. Cohen, M. P. Fahay, A. W. Kendall, \& S. L. Richardson (Eds.), Ontogeny and systematics of fishes. American Society of Ichthyologists and Herpetologists (pp. 335-354). United States: American Society of Ichthyologists and Herpetologists.

Correa-Herrera, T., \& Jiménez-Segura, L. F. (2013). Biología reproductiva de Lutjanus guttatus (Perciformes: Lutjanidae) en el Parque Nacional Natural Utría, Pacífico colombiano. Revista de Biología Tropical, 61(2), 829-840.

Correa-Rendón, J. D., Palacio, J. A., \& Jiménez-Segura, L. F. (2012). Situación actual de la pesca en el Parque Nacional Natural Utría (Pacífico Colombiano): Amenazas antrópicas para la conservación de sus recursos ícticos marinos (Tesis de Maestría). Universidad de Antioquia, Colombia.

Dingle, H. (1996). Migration: the biology of life on the move. Oxford: Oxford University Press.

Eslava, J. (1995). Colombia Pacífico, Tomo I: Climatología. Bogotá, Colombia: Proyecto Editorial del Fondo FEN.

Estupiñán, F., Von Prahl, H., \& Rubio, E. A. (1990). Ictiofauna de la Ensenada de Utría, Pacífico colombiano. Revista de Ciencias Universidad del Valle, 8, 65-75.

Escobar, J. (2010). Aspectos biológico-pesqueros de la aguja cañonera (Belonidae: Tylosurus crocodilus fodiator) en el Parque Nacional Natural Utría (Trabajo de grado). Universidad de Antioquia, Medellín, Colombia.

García, A., Claro, R., \& Koshelev, B. (1994). Reproducción. En R. Claro (Ed.), Ecología de los peces marinos de Cuba (pp. 187-262). México: Academia de Ciencias de Cuba, Instituto de Oceanografía y Centro de Investigaciones de Quintana Roo Quintana Roo.

Gómez, F., \& Vieira, C. (1996). Ictiofauna asociada a los arrecifes coralinos hermatípicos de la ensenada de Utría, Chocó, Pacífico colombiano. Universitas Scientiarium, 3(1-2), 53-61.

Liao, Y. Y., \& Chang, Y. H. (2011) Reproductive biology of the needlefish Tylosurus acus melanotus in waters around Hsiao-Liu-Chiu Island, southwestern Taiwan. Zoological studies, 50, 296-308.

Little, C., \& Kitching, J. A. (1996). The biology of rocky shores. Great Britain: Oxford University Press. 
Longart, Y., Acosta, V., Parra, B., \& Lista, M. (2012). Aspectos reproductivos del marao fósforo Hemirhamphus brasiliensis (Beloniformes: Hemirhamphidae), en la isla de Cubagua, Venezuela. Boletín del Centro de Investigaciones Biológicas, 45(1), 1-20.

Lovejoy, N. R. (2000). Reinterpreting recapitulation: systematics of needlefishes and their allies (Teleostei: Beloniformes). Evolution, 54, 1349-1362.

Myers, N., Mittermeier, R. A., \& Mittermeier, C. G., da Fonseca, G. A. B., \& Kent, J. (2000). Biodiversity hotspots for conservation priorities. Nature, 403, 853-858.

Nelson, J. S. (2006). Fishes of the World (4th ed.). Hoboken, New Jersey, USA: John Wiley \& Sons.

Nikolsky, G. (1963). The ecology of fishes. New York: Academic Press.

Palacios-Salgado, D. S., Moreno-Sánchez, X. G., AbitiaCárdenas, L. A., \& Gutiérrez-Sánchez, F. J. (2012). Ichthyodiversity of San José, San Francisquito, and El Pardito Islands in the Southwestern Gulf of California, Mexico. Acta Ichthyologica Et Piscatoria, 42(3), 177-191.

Rhodes, K. (2003). Spawning Aggregations Survey in the Federated Status of Micronesia. Federated States of Micronesia: Western Pacific Fisher Survey Series. Society for the Conservation of Reef Fish Aggregations.

Robertson, D. R., Grove, J. S., \& McCosker, J. E. (2004). Tropical transpacific shorefishes. Pacific Science, $58,507-565$.

Rojas, P. A., \& Zapata, L. A. (2006). Peces demersales del Parque Nacional Natural Gorgona y su área de influencia, Pacífico, Colombiano. Biota Colombiana, $7(002), 211-244$

Saborido, R. F. (2004). Ecología de la reproducción y potencial reproductivo en las poblaciones de peces marinos. España: Instituto de investigaciones Marinas, Universidad de Vigo.

Tobón, A., Rubio, E. A., \& Giraldo, A. (2008). Composición y análisis taxonómico de la ictiofauna del golfo de Tribugá, Pacífico norte de Colombia. Latin American Journal of Aquatic Research, 36(1), 93-104.

Unidad Administrativa Especial del Sistema de Parques Nacionales Naturales (UAESPNN). (1998). Plan de Manejo del Parque Nacional Natural Utría: Versión preliminar. Medellín, Colombia: UAESPNN.

Unidad Administrativa Especial del Sistema de Parques Nacionales Naturales (UAESPNN). (2007). Plan de Manejo 2007-2011 del Parque Nacional Natural Utría, resumen ejecutivo. Medellín, Colombia: UAESPNN.

Unidad Administrativa Especial del Sistema de Parques Nacionales Naturales -Dirección Territorial Noroccidente (UAESPNN-DTNO). (2006). Parque nacional natural Utría plan de manejo 2005-2009. Medellín, Colombia: UAESPNN.

Vazzoler, A. E. (1996). Biologia da reprodução de peixes teleósteos: teoria e prática. Maringá: Editora da universidade da Maringá.

Von Prahl, H., Cantera, J., \& Contreras, R. (1990). Manglares y hombres del Pacífico colombiano. Bogotá: Fondo FEN Colombia.

Wallace, R. A., \& Selman, K. (1981). Cellular and dynamic aspects of oocyte growth in teleosts. American Zoologist, 21, 325-343.

Williams, E. H., Jr., \& Bunkley-Williams, L. (2005). Book review: Ginger Garrison, Peces de la Isla del Coco Fishes. Instituto Nacional de Biodiversidad (INBio), Santo Domingo, Costa Rica, 2000. Reviews in Fish Biology and Fisheries, 15, 177-187.

Yáñez-Arancibia, A. (1986). Ecología de la zona costera: análisis de siete tópicos. Mexico: Editorial AGT. 
